\title{
Assessment of the nlmixr R-package for population pharmacokinetic modeling: A metformin case study
}

\author{
Wen Yao Mak ${ }^{1}$, Qing Xi Ooi ${ }^{2}$, Cintia Cruz ${ }^{3}$, Irene Looi $^{4}$, Kah Hay Yuen ${ }^{5}$, and Joseph \\ Standing 6
}

\author{
${ }^{1}$ Hospital Pulau Pinang \\ ${ }^{2}$ Pharmetheus AB \\ ${ }^{3}$ Mahidol University \\ ${ }^{4}$ Hospital Seberang Jaya \\ ${ }^{5}$ Universiti Sains Malaysia \\ ${ }^{6}$ University College London
}

November 7, 2021

\begin{abstract}
Aim: nlmixr offers first-order conditional estimation with or without interaction (FOCE or FOCEi) and stochastic approximation estimation-maximisation (SAEM) to fit nonlinear mixed-effect models (NLMEM). We modelled metformin's population pharmacokinetics with flip-flop characteristics within nlmixr framework and investigated SAEM and FOCEi's performance with respect to bias, precision, and robustness. Method: Compartmental pharmacokinetic models were fitted. The final model was determined based on the lowest objective function value and visual inspection of goodness-of-fit plots. To examine flip-flop pharmacokinetics, k_a values of a typical concentration-time profile based on the final model were perturbed and changes in the steepness of the terminal elimination phase were evaluated. The bias and precision of parameter estimates were compared between SAEM and FOCEi using stochastic simulations and estimations. For robustness, parameters were re-estimated as the initial estimates were perturbed 100-times and resultant changes evaluated. Results: A one-compartment model with transit compartment for absorption best described the data. At low n, Stirling's approximation of n! over-approximated plasma concentration unlike the log-gamma function. Flip-flop pharmacokinetics were evident as the steepness of the terminal elimination phase changed with k_a. Mean rRMSE for fixed-effect parameters was 0.932. When initial estimates were perturbed, FOCEi estimates of k_a and food effect on k_a appeared bimodal and were upward biased. Discussion: nlmixr is reliable for NLMEM even if flip-flop is present but caution should be exercised when using Stirling's approximation for $\mathrm{n}$ ! in the transit compartment model. SAEM was marginally superior to FOCEi in bias and precision, but SAEM was superior against initial estimate perturbations.
\end{abstract}

\section{Hosted file}

1_MANUSCRIPT_25102021.docx available at https://authorea.com/users/444919/articles/544551assessment-of-the-nlmixr-r-package-for-population-pharmacokinetic-modeling-a-metformincase-study

\section{Hosted file}

2_TABLE_25102021.docx available at https://authorea.com/users/444919/articles/544551assessment-of-the-nlmixr-r-package-for-population-pharmacokinetic-modeling-a-metformincase-study 

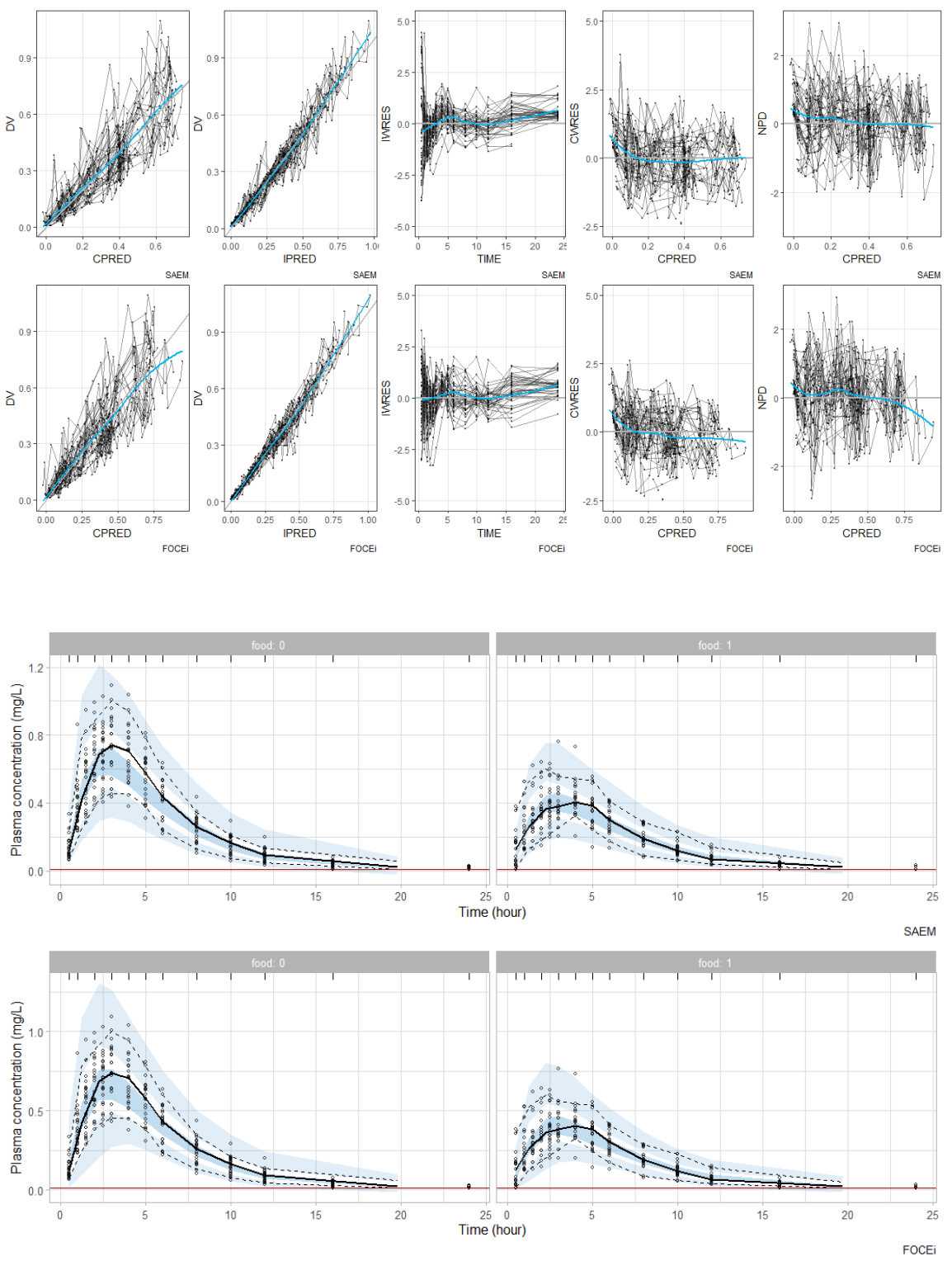

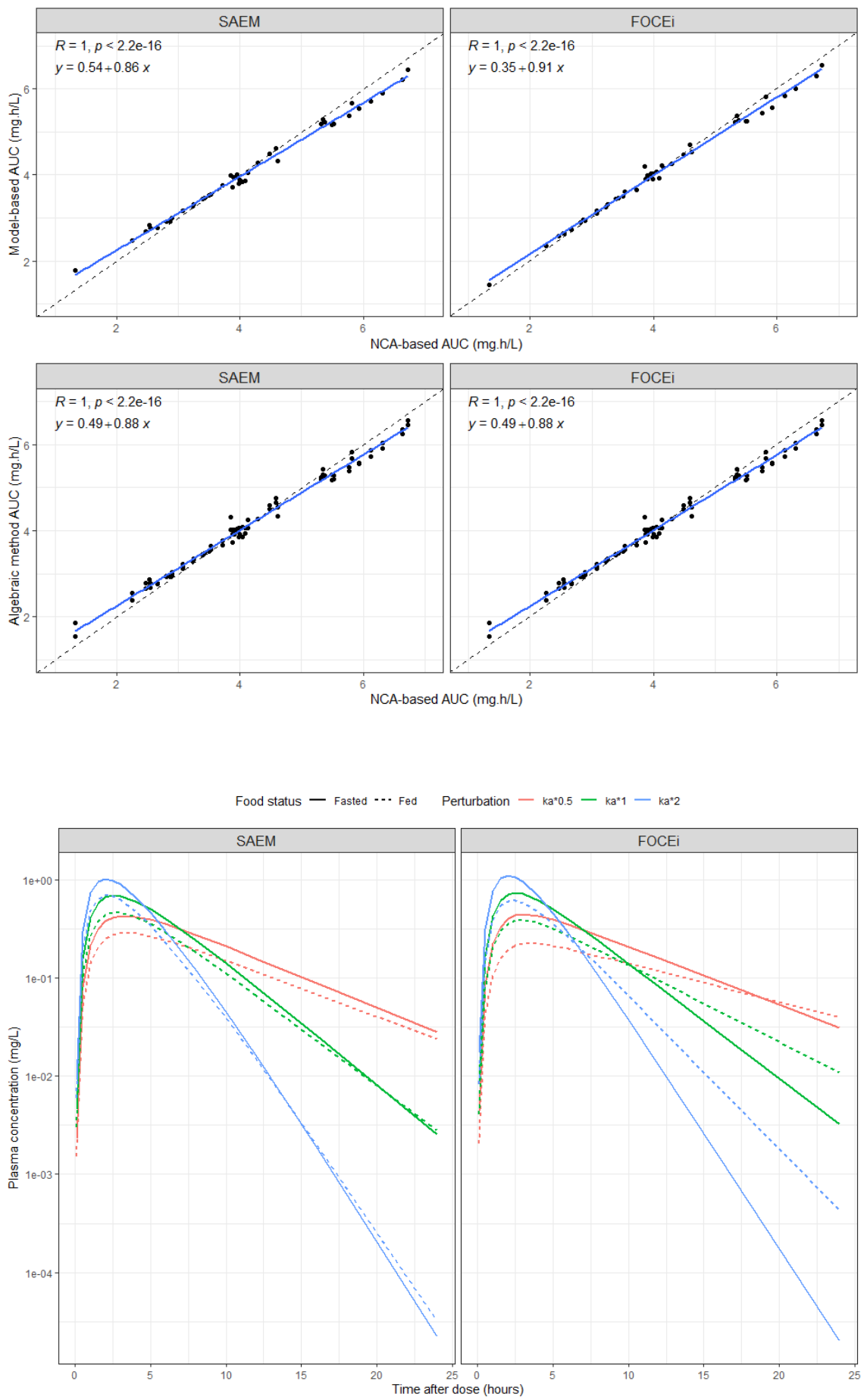

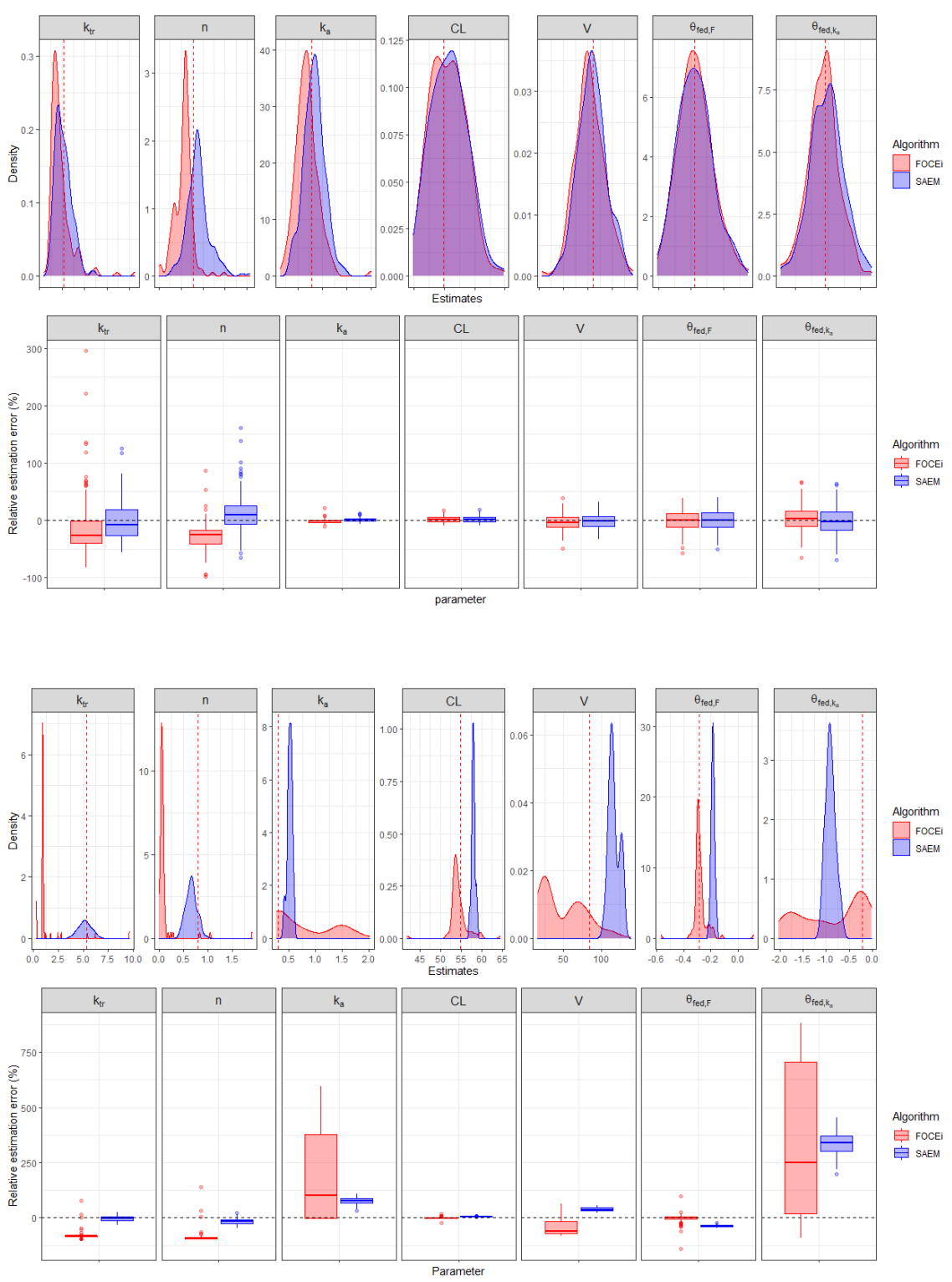